\section{Volatilisation from solid particles of the regolith}

WE have previously ${ }^{1}$ dwelt on the possible volatilisation of some elements from continuous melts of lunar surface material as a result of which it becomes depleted in elements such as $\mathrm{Na}$, $\mathrm{K}$ and $\mathrm{Rb}$, and soon. The mechanism of volatilisation proper was not confined to the liquid phase, and so they can also volatilise from the solid phase. Moreover, if solidification is not accompanied by crystallisation, the temperature dependence of the volatilisation time constant $\tau_{\mathrm{v}}$ found in ref. 1 should remain unchanged.

Table 1 Calculated values $\tau_{\mathrm{v}}$ for volatilisation of $\mathrm{Na}$ from lunar material (terrestrial time)

\begin{tabular}{ccccc}
\hline $\begin{array}{c}\text { Particle } \\
\text { size }(\mathrm{cm})\end{array}$ & 1,500 & 900 & 500 & 300 \\
1 & $15 \mathrm{~h}$ & $5 \mathrm{~d}$ & $25 \mathrm{~d}$ & $300 \mathrm{yr}$ \\
$10^{-2}$ & $0.15 \mathrm{~h}$ & $1.2 \mathrm{~h}$ & $0.25 \mathrm{~h}$ & $3 \mathrm{yr}$ \\
\hline
\end{tabular}

Table 1 gives the calculated values $\tau_{\mathrm{V}}$ for the volatilisation of $\mathrm{Na}$ (see ref. 1) from the liquid and solid lunar material. For regolith particles the value $\tau_{v}$ is negligible compared with the geological time scale since $\tau_{v} \propto d$.

In ref. 1 the assumption that the lunar surface material was liquid was used only so that the complicated process of the transport of volatile elements to the surface could be ignored. In the solid phase, however, it is just their transport to the surface that may be the weak point of the calculation of the depletion process.

In this case the transport mechanism is mainly diffusive and, hence, a simple estimate of the diffusion time constant $\tau_{d}$ can be carried out

$$
\tau_{\mathrm{d}}=\frac{d^{2}}{D} ; \quad D \sim \exp \left(-E_{\mathrm{a}} / R T_{\mathrm{s}}\right)
$$

where $D$ is the diffusion coefficient and $E_{\mathrm{a}}$ is the diffusion activation energy.

Since the dependence of $\tau_{\mathrm{v}}$ on $d$ is linear ${ }^{1}$

$$
\tau_{\mathrm{v}}=\tau_{0 \mathrm{v}} d
$$

where

$$
\tau_{\mathrm{ov}} \sim \exp \left[-(L-A) / R T_{\mathrm{s}}\right]
$$

and $(L-A)$ is the effective heat of evaporation ${ }^{1,2}$, the depletion process will be limited by volatilisation $\left(\tau_{v} \gg \tau_{d}\right)$ in the region of the smallest particles and by diffusion $\left(\tau_{d} \gg \tau_{v}\right)$ in the region of large particles. The boundary between these regions $d=d_{1}$ can be determined from the condition $\tau_{\mathrm{v}}=\tau_{\mathrm{d}}$; then

$$
d_{1}=D \tau_{0 \mathrm{v}} \sim \exp \left[-\left(E_{\mathrm{a}}-(L-A)\right) / R T_{\mathrm{s}}\right]
$$

The quantities $E_{\mathrm{a}}$ and $(L-A)$ have a similar physical meaning, and the difference between them $E_{\mathrm{s}}=E_{\mathrm{a}}-L+A$ should be close to the heat of adsorption, that is, it should be of the order of kcalorie $\mathrm{mol}^{-1}$ (ref. 2).

Using equation (1), one can show that, at all temperatures of interest $\left(250 \mathrm{~K} \lesssim T_{0} \lesssim 1,500 \mathrm{~K}\right)$, the value of $d_{1}$ lies within the region of regolith particle sizes, $\sim 10^{-4}-10^{-1} \mathrm{~cm}$, and because $E_{\mathrm{s}}>0$ it increases with decreasing temperature.

In general, putting $\tau_{0}=\max \left(\tau_{\mathrm{v}}, \tau_{\mathrm{d}}\right)$, one may assume that the rate of depletion of volatile elements from lunar material is determined by the time constant of the process 'weak point' $\tau_{0}\left(\tau_{0} \lesssim 10^{4} \mathrm{yr}\right.$ for $d \lesssim 10^{-1} \mathrm{~cm}$ and $\left.250 \mathrm{~K} \lesssim T_{\mathrm{s}} \lesssim 400 \mathrm{~K}\right)$. This interval corresponds approximately to the temperature

variations, $T_{\mathrm{s}}$, of the upper layers of the regolith during the lunar day.

Since volatilisation and diffusion occur only in the day, and are almost totally switched off at night, the effective value $\tau_{0}$ for the lunar day does not increase more than twice.

Next, it should be kept in mind that under meteoroid bombardment, and some other factors, the regolith particles, at least those from the uppermost layer $\left(h \simeq 10^{-1} \mathrm{~cm}\right)$, plunge into the lower ( $h \lesssim 1 \mathrm{~cm}$ ) layers and then rise to the surface, intermixing at the rate $U_{\mathrm{p}} \simeq 1 \mathrm{~cm} / 10^{6} \mathrm{yr}$ (ref. 3). On the other hand, it is known ${ }^{3,4}$ that the rate of regolith formation is $U_{\mathrm{f}} \simeq 10^{-1} \mathrm{~cm} / 10^{6} \mathrm{yr}$.

Thus, for the regolith upper layer $\left(h \simeq 10^{-1} \mathrm{~cm}\right)$ one has the following inequality

$$
\tau_{0} \ll \tau_{p} \ll \tau_{f}
$$

where $\tau_{0} \lesssim 10^{4} \mathrm{yr}, \tau_{\mathrm{p}}=h / U_{\mathrm{p}} \simeq 10^{5} \mathrm{yr}$, and $\tau_{\mathrm{f}}=h / U_{\mathrm{f}} \simeq 10^{6}$ $\mathrm{yr}, \tau_{p}$ and $\tau_{f}$ being the time constants of the corresponding processes.

The relationship between $\tau_{\mathrm{p}}$ and $\tau_{\mathrm{f}}$ shows that each regolith particle of a given layer was on the surface $>10$ times whereas the first inequality in equation (2) implies that even a single sojourn of a particle on the surface is sufficient for the particle material to be significantly depleted in volatile elements.

Earlier we have shown theoretically and experimentally ${ }^{1.5}$ that the process of volatilisation from the liquid phase as a result of meteoroid impacts is inessential because the volume (size) of each melt is small, and the melts solidify very rapidly. These two factors also rule out the chemical fractionation of the lunar regolith by impact melting, proposed in ref. 6 .

Meteoroid bombardment, however, can have a decisive effect on the process of volatilisation of $\mathrm{Na}, \mathrm{K}$ and other elements from solid particles in the regolith, crushing and intermixing them. If this process really occurred (and occurs at present) on the Moon, then a chemical analysis of lunar rocks of the appropriate sizes could reveal the presence of a volatileelement concentration gradient.

Finally it should be noted that the approach we suggest may be also useful for solving some terrestrial ecological problems like that in ref. 7.

We are grateful to Dr G. A. Leikin for stimulating discussion on this subject.

Institute for Space Research, USSR Academy of Sciences, Moscow, USSR

YU. B. CHERNYAK

M. D. NUSSINOV

Received May 17; accepted September 10, 1976.

Nussinov, M. D., and Chernyak, Yu. B., Nature, 261, 666 (1976); The Moon 13, 4. 377 (1975).

2 Cubo, R., Thermodynamics (North-Holland, Amsterdam, 1968),

Hörz, F., et al., in The Chemistry of the Moon and Planets (Proceedings Soviet American Conference on the Chemistry of the Moon and Planets), M (Nauka, 1975).

4 Gold, T., in The Moon (edit, by Runcorn, S. K., and Urey, H. C.), 52-66 (IAU Symposium, D. Reidel Publishing Company, Dordrecht, Holland. 1972).
Bachtina, A. P., Nussinov, M. D.. Feldman, V. I., and Chernyak, Yu. B., Vest. mosk. gos. univ. (ser. geol.), 3 (1976).

Adams, J. B., Charette, M.P., and Michael, R. J., Science, 190, 4212 (1975) Goldberg, E. D., Nature, 260, 128 (1976).

\section{Concentrations of dissolved copper in the eastern Atlantic Ocean $23^{\circ} \mathrm{N}$ to $47^{\circ} \mathrm{N}$}

Considerable uncertainties still exist as to the abundances of most of the trace metals in seawater, including some of the more intensively investigated elements. Recent analyses of copper in surface waters south of New Zealand ${ }^{2}$ and in waters from various depths over the East Pacific Rise ${ }^{2}$ have given values of $0.06-0.40 \mu \mathrm{g} \mathrm{l}^{-1}$, and a similar range has been found by $\mathbf{M}$. J. McCartney (personal communication) for samples from the Mediterranean Sea and the northeastern Atlantic Ocean. A geometric mean concentration of $0.26 \mu \mathrm{g} \mathrm{l}^{-1}$ has been reported for samples collected off the shelf west of Scotland ${ }^{3}$. These values are either below 